\title{
Recognition of Reviewers and Associate Editors
}

Reviewing manuscripts is an essential part of scientific research evaluation. Each volunteered manuscript accepted for publication is review by three peers, an associate editor, and the editor. The objective of reviewer selection is to obtain assistance from scientists who may know the manuscript topic better than the associate editor or editor.

The reviewers primary responsibilities are to evaluate the scientific quality and the accuracy of data interpretation and to help the author to improve the manuscript. Below is a list of WSSA members and other individuals who have reviewed one or more manuscripts for Weed Technology between October 19, 1994 and November 8, 1995, and whose reviews have been returned to me. Others assigned during this same period are still pending and will be reported next year. Thanks to these colleagues for helping to maintain and to enhance the weed science profession. Please forgive any errors or omissions.

Thanks also to Associate Editors Randy L. Anderson, Thomas A. Bewick, Barry J. Brecke, Douglas Buhler, John Cardina, William J. Chism, William W. Donald, Joan Dusky, Jerry M. Green, Neil Harker, Robert G. Harvey, Jr., George Kapusta, James R. Martin, Robert F. Norris, Thomas F. Peeper, Donald Penner, Edward P. Richard, Phillip Stahlman, Clarence J. Swanton, William H. VandenBorn, Leslie Weston, Gail Wicks, John Wilcut, and Alan C. York; to Technical Editor Leanne D. Mitchell; and to the WSSA headquarters staff.

Chester L. Foy, Editor

Abbas, H. K.

Abernathy, J. R. Ahrens, W. H.

Appleby, A. P.

Ball, D. A.

Banks, J. C.

Banks, P. A.

Barrentine, W. L.

Barrett, M.

Bayer, D. E.

Becker, R.

Beckett, T. H.

Bellinder, R.

Beste, C. E.

Bhowmik, P. C.

Billet, D.

Blackshaw, R. E.

Bode, $L$.

Boerboom, C.

Borman, M. M.

Bowes, G. G.

Boyd, J.

Boydston, R.

Boyetchko, S.

Boyette, C. D.

Branham, B.

Brown, S. M.
Buhler, D.

Burnside, O. C.

Burow, R.

Burrill, L. C.

Byrd, J. D., Jr.

Callahan, L. M.

Callihan, R. H.

Camper, N. D.

Cardina, J.

Castello, J.

Chilton, W. S.

Coats, G. E.

Coble, $\mathrm{H}$.

Corbin, F. T.

Crabtree, G.

Curran, W. S.

Czapar, G.

Darwent, A. L.

Dawson, J. H.

DeFelice, M. S.

Derr, J.

Devine, M. D.

Devlin, D. L.

Devlin, R. M.

Dewey, S. A.

Dexter, A.
Doll, J.

Donald, P. A.

Donald, W. W.

Doohan, D. J.

Dowler, C. C.

Eberlein, C.

Elmore, C. D.

Elmore, C. L.

Eplee, R. E.

Erickson, C. G.

Eskridge, $\mathrm{K}$.

Evans, R. R.

Everitt, J. H.

Fales, S. L.

Forcella, F.

Frans, R. E.

Friesen, M. L.

Fuerst, E. P.

Gealy, D.

Gilliam, C. H.

Glenn, S.

Gogan, G.

Gossett, B. J.

Green, J. M.

Green, R. L.
Gregory, M.

Gressel, J.

Grichar, W. J.

Griffin, J. L.

Gronwald, J.W.

Gunsolus, J. L.

Guy, C. B.

Hageman, L.

Hagood, E. S., Jr.

Hall, D. W.

Hall, M. R.

Hanks, J. E.

Hanson, R.

Harris, P.A.

Harrison, H. F., Jr.

Harrison, $\mathrm{K}$.

Hart, S. E.

Hartwig, N. L.

Hartzler, R. G.

Harvey, R. G., Jr.

Hatzios, K. K.

Hayes, $\mathbf{R}$.

Helms, R. S.

Henderson, J.

Hickman, M.V.

Holm, F. A.

Holm, L. G. 


\begin{tabular}{|c|c|c|c|}
\hline Holt, J. S. & Lyon, D. J. & Patterson, D. T. & Street, J. \\
\hline Hopen, H. J. & & Penner, D. & Street, J. E. \\
\hline Horak, M. J. & Manthey, F. & Peters, A. & Stritzke, J. F. \\
\hline Huddleston, E. W. & Martin, A. R. & Peterson, D. E. & \\
\hline Humke, K. & Masiunas, J. B. & Pirie, W. & Teasdale, J. R. \\
\hline Hunter, J. H. & $\begin{array}{l}\text { Maxwell, B. } \\
\text { McCarty, L. D. }\end{array}$ & Porter, W. C. & $\begin{array}{l}\text { Templeton, G. E. } \\
\text { Thill, D. C. }\end{array}$ \\
\hline Ivany, J. A. & $\begin{array}{l}\text { McMullan, P. } \\
\text { Mersie, W. }\end{array}$ & Quimby, P. C., Jr. & Tuellar, P. T. \\
\hline Jeffery, L. S. & Messersmith, C. G. & Rardon, P. L. & VandenBorn, W. H. \\
\hline Johnson, B. J. & Miller, S. D. & Regnier, E. E. & Vaughn, K. C. \\
\hline Johnson, D. R. & Mitich, L. W. & Renner, K. A. & Vidrine, P. R. \\
\hline Johnson, W. C., II & Monaco, T. J. & Reynolds, D. B. & 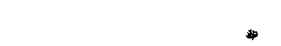 \\
\hline Johnson, W. G. & Monks, C. D. & Rhodes, G. N., Jr. & Walker, R. H. \\
\hline Jordan, D. L. & Monks, D. W. & Rioux, R. & Wall, D. \\
\hline Jordan, T. N. & $\begin{array}{l}\text { Morrison, I. } \\
\text { Morton, C. }\end{array}$ & $\begin{array}{l}\text { Riveland, N. R. } \\
\text { Roeth, F. W. }\end{array}$ & $\begin{array}{l}\text { Wauchope, R. D. } \\
\text { Wax, L. M. }\end{array}$ \\
\hline Keeling, J. W. & Moser, L. E. & & Weaver, S. \\
\hline Kells, J. J. & Mosier, L. J. & Saari, L. & Wehtje, G. \\
\hline Kendig, J. A. & Moyer, J. R. & Schreiber, M. M. & West, C. P. \\
\hline King, D. L. & Mueller-Warrant, G. & Schroeder, J. & Westerman, R. B. \\
\hline Klein, R. N. & Mullins, D. & Schweizer, E. E. & Westra, P. \\
\hline Klett, J. E. & Murdock, E. C. & Shaner, D. L. & Westwood, J. H. \\
\hline Kremer, R. J. & Murray, D. S. & Sharma, M. P. & Whitson, T. \\
\hline Kuhns, L. & & Shaw, D. & Wiese, A. \\
\hline Kunkel, D. L. & $\begin{array}{l}\text { Nalewaja, J. D. } \\
\text { Nash, R. G. }\end{array}$ & $\begin{array}{l}\text { Shea, P. J. } \\
\text { Sims, B. D. }\end{array}$ & $\begin{array}{l}\text { William, R. D. } \\
\text { Williams, R. D. }\end{array}$ \\
\hline Langeland, K. A. & Neal, J. C. & Skipper, H. D. & Wilson, H. P. \\
\hline Lanini, W. T. & & Smeda, R. J. & Wilson, R. G. \\
\hline Lee, R. D. & ODonovan, J. & Snipes, C. E. & Witt, W. W. \\
\hline Legere, A. & Ogg, A. G. & Solie, J. B. & Wixson, M. \\
\hline Lencse, R. J. & Oliver, L. R. & Srejcar, T. & \\
\hline Lewis, W. M. & Orcutt, D. M. & Stahlman, P. & York, A. C. \\
\hline $\begin{array}{l}\text { Loeppky, H. A. } \\
\text { Lym, R. G. }\end{array}$ & Owen, M.D.K. & $\begin{array}{l}\text { Stoller, E. W. } \\
\text { Stoltenberg, D. E. }\end{array}$ & Young, F. L. \\
\hline
\end{tabular}

